\title{
Of fingers, toes and penises
}

V ertebrate Hox genes are essential for limb development. The posterior-most Hoxd and Hoxa genes are required for growth and patterning of digits and are also strongly expressed in the genital bud, which gives rise to the urogenital system, including the penis. Here, we show that removal of posterior Hox gene function results in a concomitant loss of digits and genital budderivatives, illustrating that similar developmental mechanisms are at work in these different buds.

We raised compound mutant mice carrying the hypodactyly $(H d)$ allele $^{1}$ and null alleles of the HoxD complex inactivating either Hoxd-13 (ref. 2), or Hoxd-11, Hoxd-12 and Hoxd-13 in cis configuration $H o x D^{D e l}$ (ref. 3). Hd mice have a deletion in Hoxa-13 (ref. 1), so these combinations removed most Hox gene function in the digits. The effect was comparable to that observed when using a Hoxa-13 loss-offunction allele ${ }^{4}$ a total digit agenesis (Fig. 1a). Double-mutant limbs were truncated right after the zeugopods (Fig. 1a; large arrowheads) with only traces of proximal tarsal condensations (Fig. 1a).

Expression of Hoxd genes was visualized using a lac $Z$ reporter gene inserted either in Hoxd-13 (Fig. 1b) or at the HoxD $D^{D e l}$ locus ${ }^{3}$. $\beta$-gal staining revealed a sizeable blue eminence in control $\mathrm{Hd} /+$; Del/+ mice (Fig. 1c) which showed considerable digit development. But a second $\mathrm{Hd}$ allele $(\mathrm{Hd} / \mathrm{Hd}$; $\mathrm{Del} /+$ mice) induced a severe truncation of the eminence (Fig. 1d). Removing group-13 function $\left(\mathrm{Hd} / \mathrm{Hd}\right.$; Hoxd-13 $\left.{ }^{-/-}\right)$led to complete agenesis of the bud (Fig. 1e, f). This progressive reduction in genital bud size with reductions in Hox gene dose was accompanied by proportional reductions in digit size (compare Fig. 1a with c, d). Mild anatomical defects in these two structures also occur in the human hand-foot-genital syndrome which involves a mutation within Hoxa-13 (ref. 5).

Histological analyses of $\mathrm{Hd} / \mathrm{Hd}$; Hoxd $-13^{-/-}$fetuses confirmed the complete absence of external genitalia (Fig. 1g, h). In addition, the lower urinary system, particularly the bladder and urethra, were missing, as well as derivatives of the urogenital sinus. Kidneys, which do not express Hoxd-13 (ref. 6), were present in compound mutant animals. $\mathrm{Hd} / \mathrm{Hd}$;Hoxd $-13^{-1-}$ fetuses died in utero before embryonic day 16 like most $\mathrm{Hd} / \mathrm{Hd}$ mice ${ }^{1}$.

The development of the distal limbs and genital eminence share important features. Both structures are regions of apical growth and involve epithelial-mesenchymal interactions. Further, digits and genitalia represent 'morphogenetic ends' of the body, digits

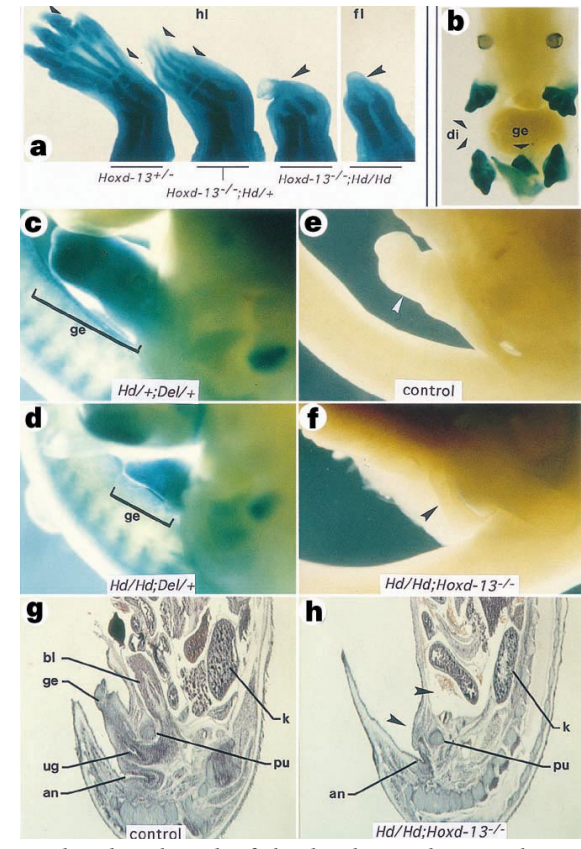

at the distal end of the limbs and genitalia at the trunk posterior. Molecular data support this analogy, as most genes expressed during digit development are also functional in the genital eminence. Hox genes are particularly relevant as 5'-located Hoxa and Hoxd genes are involved in organizing the terminal parts of axial structures ${ }^{5-7}$

There is other evidence to suggest a phylogenetic link between digits and external genitalia. In fish, which lack digits and external genitalia, posterior Hox genes are expressed in areas from which these structures emerged (the cloacal region and paired fins). Also, Hoxd gene expression in developing murine digits and genital eminence is controlled by the same transcriptional enhancer element ${ }^{8-10}$. Finally, Hoxc-13 and Hoxb-13, the other group-13 paralogues, are expressed neither in digits nor in the genital bud ${ }^{11,12}$.

During evolution, the acquisition of the distal Hoxd expression domain in paired fin buds may have been linked to the emergence of digits by extending the terminal phase of proliferation ${ }^{13}$. Related phylogenetic histories between digits and the penis/clitoris thus seem plausible and may reflect combined adaptive or preadaptive solutions to the transition towards a terrestrial environment. Palaeontological observations do not exclude the possibility that ancestral tetrapods had protruding external genitalia. As such animals were aquatic ${ }^{14}$, a genital organ may not have been linked to terrestrial life but instead might have reflected the requirement for elaborate internal fertilization.

Digits and an ancestral genital organ could have emerged from the same genetic
Figure 1 Absence of digits and genitalia in mice lacking posterior Hox function. a, Dissected hindlimbs (hl) and forelimbs (fl) from control mice and mice lacking most or all group-13 function. Small arrowheads, digits; large arrowheads, absence of digit. b, Expression of Hoxd-13/lacZ reporter gene in digits (di) and genital eminence (ge) of a 12.5-day-old fetus (E12.5). c, Expression of Hoxd/lacZ reporter in E13.5 developing genital eminence of fetuses with a phenotype close to normal $(\mathrm{Hd} /+$; Del/+). d, Removal of one dose of Hoxa-13 ( $\mathrm{Hd} / \mathrm{Hd}$; Del/t+) led to truncation of the genital eminence. Left hindlimbs were removed to view the eminence. Other blue domains are sites of posterior HoxD locus expression. e, Control E15.5 fetus with genital eminence (arrow). $\mathbf{f}$, Same-aged fetus in absence of group-13 function. $\mathbf{g}, \mathbf{h}$, Mid- to parasaggital sections through fetuses shown in $\mathbf{e}$ and $\mathbf{f}$, respectively. ug, urogenital sinus; an, anus; k, kidneys; pu, pubis; bl, bladder. In the absence of group-13 function, the eminence is absent as well as the bladder (arrows). The rectum and anus are also abnormal (arrow).

regulatory innovation. It is nevertheless equally possible that the underlying molecular mechanisms were first deployed in forming an external genital organ and subsequently used to produce digits, or vice versa. In any event, the combined improvement of apical growth mechanisms along these axes produced the adaptative potentials necessary for efficient locomotion and internal fertilization in the terrestrial environment.

Takashi Kondo, József Zákány Jeffrey W. Innis, Denis Duboule Department of Zoology and Animal Biology, University of Geneva, Quai Ernest Ansermet 30, 1211 Geneva 4, Switzerland and Departments of Human Genetics and Pediatrics,

University of Michigan Medical School,

Ann Arbor, Michigan 48109-0618, USA e-mail:duboule@sc2a.unige.ch

1. Mortlock, D. P., Post, L. C. \& Innis, J. W. Nature Genet. 13 284-289 (1996)

2. Dollé, P. et al. Cell 75, 431-441 (1993).

3. Zákány, J. \& Duboule, D. Nature 384, 69-71 (1996).

4. Fromental-Ramain, C. et al. Development 122, 2997-3011 (1996) 5. Mortlock, D. P. \& Innis, J. W. Nature Genet. 15, 179-180 (1997) 6. Dollé, P., Izpisúa-Belmonte, J.-C., Brown, J. M., Tickle, C. \& Duboule, D. Genes Dev. 5, 1767-1776 (1991)

7. Kondo, T., Dollé, P., Zákány, J. \& Duboule, D. Development 122, 2651-2659 (1996)

8. van der Hoeven, F., Zákány, J. \& Duboule, D. Cell 85, 1025-1035 (1996).

9. Hérault, Y., Fraudeau, N., Zákány, J. \& Duboule, D. Development 124, 3493-3500 (1997).

10. Peichel, C. L., Prabhakaran, B. \& Vogt, T. F. Development 124, 3481-3492 (1997).

11. Peterson, R. L., Papenbrock, T., Davda, M. M. \& Awgulewitsch, A. Mech. Dev. 47, 253-260 (1994).

12. Zeltser, L., Desplan, C. \& Heintz, N. Development 122, 2475-2484 (1996).

13. Sordino, P., van der Hoeven, F. \& Duboule, D. Nature 375, 678-681 (1995).

14. Coates, M. I. Development (suppl.) 169-180 (1994). 